\title{
Liver Metastasis of Hepatoid Colonic Adenocarcinoma: A Rare and Unusual Entity With Poor Prognosis and Review of the Literature
}

\author{
Ming $\mathrm{Hu}^{\mathrm{a}}$, Weidong Liu ${ }^{\mathrm{b}, \mathrm{d}}$, Feng Yin ${ }^{\mathrm{a}}$, Dongwei Zhang ${ }^{\mathrm{c}}$, \\ Xiuli Liu ${ }^{\mathrm{a}}$, Jinping Lai ${ }^{\mathrm{a}, \mathrm{d}}$
}

\begin{abstract}
Hepatoid adenocarcinoma (HAC) is rare and was first reported as $\alpha$-fetoprotein (AFP)-producing tumor. It is an important variant of extrahepatic adenocarcinoma with clinicopathological presentation mimicking hepatocellular carcinoma and carries exceedingly poor prognosis. HAC most commonly originates in the stomach, and less commonly in the ovary, esophagus, lung, among other organs. HAC originating in the colon is exceedingly rare. Here we report such a case of a 63-year-old man presented as decompensated liver failure with jaundice and weakness. Computed tomography (CT) imaging findings showed multiple lesions in the liver with ascites and descending colonic mass suspicious for malignancy. The flexible sigmoidoscopy showed a $1.5 \mathrm{~cm}$ mass in the descending colon, and biopsy showed superficial fragments of tubular adenoma, but could not exclude deep invasive carcinoma. A liver biopsy was performed and showed a carcinoma with morphologic features resembling hepatocellular carcinoma. The tumor cells were positive for glypican-3, MOC31, CDX2, SATB2 and CK20, negative for arginase-1, p63, synaptophysin and chromogranin. Ki-67 highlighted $80 \%$ of the tumor cells. The pathology diagnosis was liver with metastatic hepatoid adenocarcinoma consistent with colonic primary. The patient experienced a rapid worsening of his liver function and died 3 weeks later of hepatic failure without any surgery and chemotherapy. A subsequent literature review of the 17 reported cases of HAC showed that this type of cancer frequently metastasizes to the liver with an astonishingly poor prognosis with eight patients died of the disease in less than 5 months after the diagnosis was made. Radical surgery followed by adjuvant
\end{abstract}

Manuscript submitted September 20, 2018, accepted October 22, 2018

a Department of Pathology, Immunology, and Laboratory Medicine, College of Medicine, University of Florida, Gainesville, FL, USA

bDepartment of Surgery, Xiangya Hospital, Central South University, Changsha, Hunan, China

'Department of Pathology and Laboratory Medicine, University of Rochester Medical Center, Rochester, NY, USA

${ }^{\mathrm{d} C o r r e s p o n d i n g ~ A u t h o r: ~ W e i d o n g ~ L i u, ~ D e p a r t m e n t ~ o f ~ S u r g e r y, ~ X i a n g y a ~ H o s-~}$ pital, XiangYa School of Medicine, Central South University, Changsha, 410008, China. Email: weidong.liu@csu.edu.cn; Jinping Lai, Department of Pathology, Immunology, and Laboratory Medicine, University of Florida College of Medicine, Gainesville, FL 32610, USA. Email: jinpinglai@ufl.edu

doi: https://doi.org/10.14740/gr1097 chemotherapy with chemotherapy regimen used for colorectal cancer or primary hepatocellular carcinoma may be the treatment option for colorectal HAC.

Keywords: Hepatoid colonic adenocarcinoma; Liver metastasis; Poor prognosis; DNA mismatch repair protein; Immunohistochemistry; Next generate sequencing

\section{Introduction}

Hepatoid adenocarcinoma (HAC) is a primary extrahepatic carcinoma of non-germ cell origin showing morphological features of hepatocellular differentiation. HAC is described as being morphologically reminiscent of a hepatocellular carcinoma, including nests and trabecular structure of polygonal atypical cells with abundant eosinophilic granular cytoplasm. $\mathrm{HAC}$ has been reported in multiple organs, mainly in the stomach and ovary as well as in other sites. HAC originating from colon is very rare. To date, only 17 cases of colorectal cancer with hepatoid features have been reported in English literature, and this type of cancer frequently metastasizes to the liver and often exhibits an astonishingly poor prognosis. In a disease that is too rare to anticipate results from large clinical trials to guide practice, it is important to recognize its pathological features due to its aggressive behavior. In this study, we report an extremely rare case of liver metastasis of colorectal HAC with literature review of the pathological feature of the previously published 17 cases of HAC.

\section{Case Report}

A 63-year-old male patient presented to an outside hospital and was transferred to our emergency department (ED) for concerns of decompensated liver failure. The patient had been having progressively worsening abdominal distention and swelling of his bilateral lower extremities, jaundice, and dark urine for the past 2 months. The patient reported some constipation for the last 3 - 4 months, 2 - 3 bowel movements per week, intermittent bright red blood on the toilet paper, fatigue, and unintentional weight loss of 50 pounds over the 


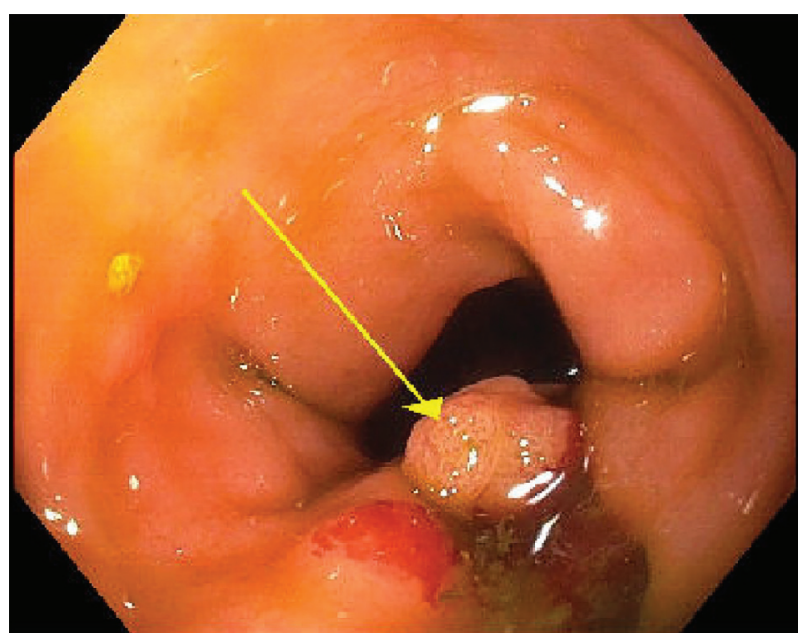

Figure 1. Endoscopic appearance of the descending colon mass (1.5 $\mathrm{cm})$.

last 6 months. His past medical history included arthritis and hypertension, and he denied any history of liver diseases. He was not a smoker, with no history of alcohol use and drug use. An outside computed tomography (CT) scan of his abdomen and pelvis was performed and revealed a mass of the splenic flexure, with multiple lesions in the liver with ascites, concerning for metastatic colon cancer. His stomach was unremarkable. In our hospital, the patient had abnormal liver function tests including increased total bilirubin $(5.7 \mathrm{mg} / \mathrm{dL}$, with direct bilirubin $3.5 \mathrm{mg} / \mathrm{dL}$ ), alkaline phosphatase (241 IU/L), and aspartate aminotransferase (AST) (194 IU/L) and normal alanine aminotransferase (ALT) (28 IU/L). Paracentesis was performed twice to remove more than $6 \mathrm{~L}$ fluids at the first time, and $10 \mathrm{~L}$ fluids 3 days later to relieve dyspnea. Flexible sigmoidoscopy showed a $1.5 \mathrm{~cm}$ polypoid mass (Fig. 1) of the descending colon, biopsy was performed and showed superficial fragments of tubular adenoma but could not exclude a deep invasive carcinoma. Interventional radiologist performed ultrasound-guided biopsy of the liver.

The liver specimen received by our department consisted of three pink-white to yellow-white soft tissue cores ranging from 1.0 to $1.3 \mathrm{~cm}$ in length, and averaging less than $0.1 \mathrm{~cm}$ in diameter. Sections of the biopsy showed three cores of poorly differentiated carcinoma in a background of fibrosis. No adjacent hepatic parenchyma was identified. The tumor cells were arranged in a trabecular pattern with round to ovoid nuclei, some with intranuclear pseudoinclusions and prominent nucleoli, and abundant eosinophilic cytoplasm resembling the morphology of a hepatocellular carcinoma (Fig. 2a, b). Immunostains were performed and the tumor cells were focally positive for glypican-3 (Fig. 2c), negative for arginase-1, p63, synaptophysin and chromogranin, and with $80 \%$ of Ki-67 labeling in tumor cells (Fig. 2d). Additional immunostains showed that the tumor cells were positive for MOC31 (Fig. 3a), CK20 (Fig. 3b), CDX2 (Fig. 3c), and SATB2 (Fig. 3d), and intact expression of mismatch repair (MMR) proteins including MLH1, MSH2, MSH6, and PMS2. Based on the morphological and immunohistochemical staining profile, the tumor was most consistent with a metastatic poorly differentiated colonic adenocarcinoma with hepatoid features.

Molecular testing of the biopsy liver mass did not display any detectable microsatellite instability (MSI) using a panel of microsatellite marker BAT25, BAT26, NR-21, NR-24, and MONO-27 [1]. The tumor was interpreted as being microsatellite stable (MSS). MSS results were consistent with intact expression of four MMR proteins by immunohistochemistry.

Next generation sequencing (NGS) was performed on the adenocarcinoma in the liver biopsy. Pathogenic mutations in
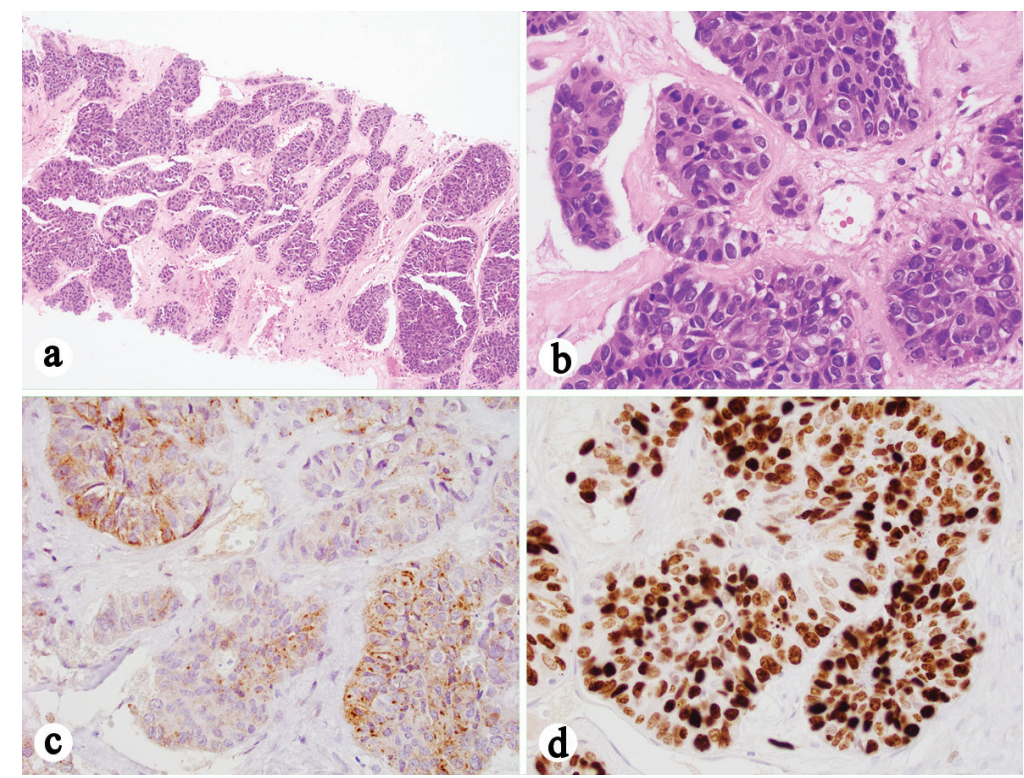

Figure 2. Liver core biopsy showing a poorly differentiated carcinoma with hepatoid features. (a) H\&E stain, $100 \times$. (b) H\&E stain, $400 \times$. (c) Immunohistochemistry showing that the tumor cells are focally positive for glypican $3(400 \times)$. (d) Ki-67 highlights $80 \%$ of the tumor cells $(400 \times)$. 


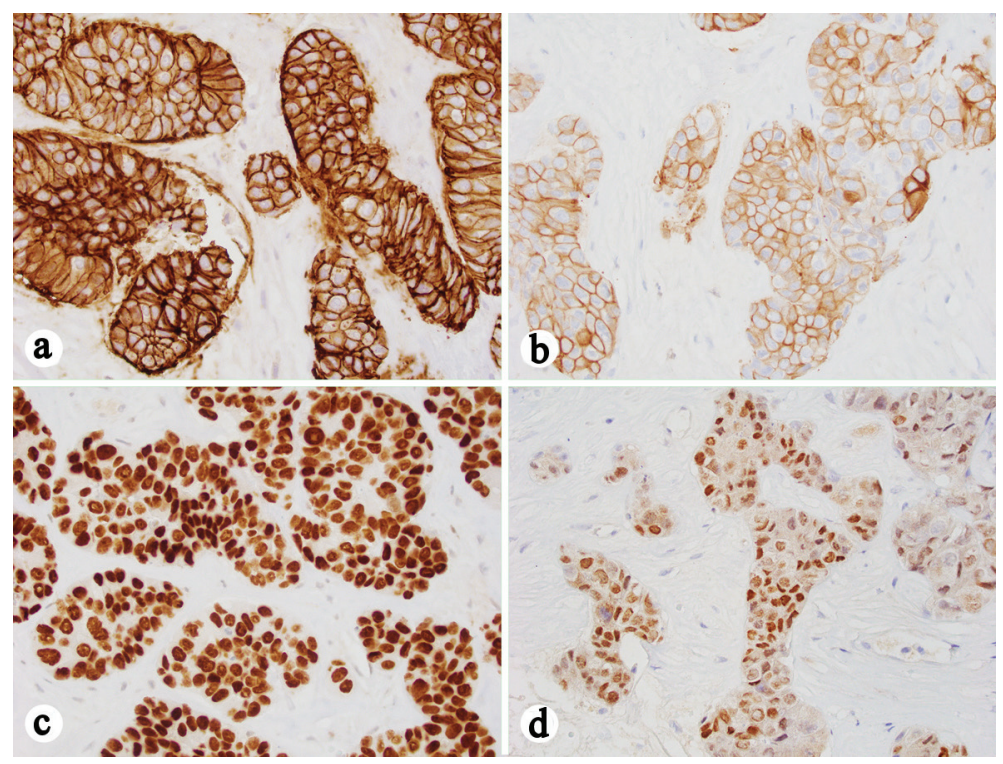

Figure 3. Immunohistochemistry showing that the tumor cells are positive for MOC-31 (a), CK20 (b), CDX2 (c) and SATB2 (d) $(a-d, 400 \times)$.

KRAS (c.38_39delGCinsAT p.G13D) and TP53 (c.743G $>$ A p.R248Q) were present. Mutations in other genes including NOTCH1, NF1, ASXL1, FLT3, U2AF2, NKL1 and JAK2 were also present.

Unfortunately, the patient experienced a rapid worsening of his liver function and 1 week later his total bilirubin was increased to $16 \mathrm{mg} / \mathrm{dL}$ with direct bilirubin $9 \mathrm{mg} / \mathrm{dL}$ and alkaline phosphatase 399 IU/L and thus he was not a candidate for chemotherapy. The patient was referred to palliative care where he elected for the arrangement of hospice care, and the patient died of liver failure 3 weeks later after the diagnosis was made.

\section{Discussion}

HAC was first reported as $\alpha$-fetoprotein (AFP)-producing tumor by Bourreille et al in 1970 [2]. Since then, HAC has been reported in multiple organs, mainly in the stomach, and less commonly in other organs including ovary, esophagus, lung, gallbladder, pancreas [3], bladder [4], uterus and colon. Colorectal HAC is extremely rare, so far, only 17 HACs have been reported in the English literature through PubMed search, and the prognosis of colorectal HAC was significantly worse than that of HAC of the stomach [5].

In this study, we report one case of HAC and further reviewed the clinicopathological characteristics of the previously published 17 patients with colorectal HAC (Table 1, [6-22]). Colorectal HAC commonly occurs in middle age to old patients (median age: 54 years; range: 36 - 75) and shows a predilection for the male sex. There were 12 male $(66.7 \%)$ and six female $(33.3 \%)$ patients with a male to female ratio of $2: 1$. The majority of patients $(14 / 15,93.3 \%)$ had elevated serum AFP levels; 16 cases showed AFP positivity by immunohistochemistry, which is helpful for an objective diagnosis.
However, an absence of AFP overproduction did not exclude a diagnosis of HAC and the capacity of tumor tissue to produce AFP had no impact on prognosis. In our case, neither serum AFP level nor immunohistochemistry for AFP on liver biopsy was performed.

An early diagnosis and treatment are crucial to improve the survival of patients with colorectal HAC. Unfortunately, a preoperative diagnosis of HAC is exceedingly difficult. First, elevated serum AFP is not a universal marker although it increases in most cases. Secondly, even preoperative biopsy is performed and it may not result in a definitive diagnosis of HAC. A definitive diagnosis of HAC depends on the histomorphological features plus immunophenotypical evidences. Histomorphological features of HAC include large and polygonal hepatocyte-like cells with intense eosinophil cytoplasm and big centrally located nucleus. Diagnosing metastatic HAC involving the liver may be particularly challenging due to its hepatoid appearance in the liver biopsy. Identifying the HAC in other sites as primary is essential for such a diagnosis. In our case, outside CT scan revealed a mass in the colon, with multiple lesions in the liver, though flexible sigmoidoscopy showed a polyp in the colon, and biopsy showed superficial fragments of tubular adenoma; as we could not exclude deep invasive carcinoma, we still have to analyze the pathological features of the liver masses, and primary HCC must be ruled out. In our case, glypican-3, a marker of hepatocellular differentiation, was focally positive. Glypican-3 was reported to be $100 \%$ positive by $\mathrm{Su}$ et al in a HAC literature review [5]. Arginase-1, a novel sensitive and specific marker for hepatocellular carcinoma (HCC) [23], was negative in our case. In addition, the tumor cells in our case were reactive to anti-MOC31 antibodies which is a marker of adenocarcinoma, but usually do not react with hepatocytes [24]. The previously mentioned combination of histopathologic and immunohistochemical evidences help differentiate metastatic adenocarcinoma to the liver from 
Table 1. Clinical Features of Reported Cases of Hepatoid Colorectal Carcinomas

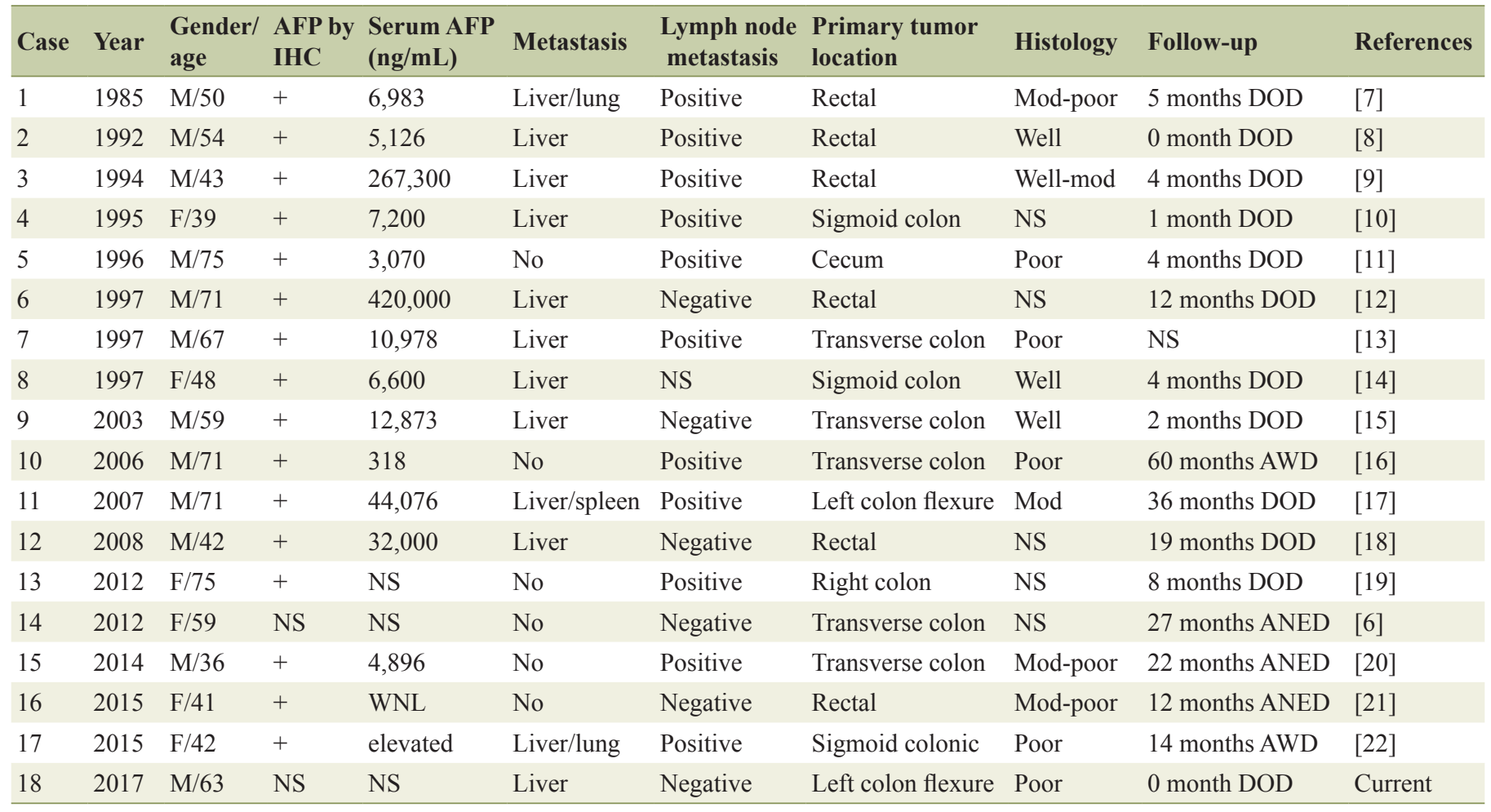

IHC: immunohistochemistry; Well: well differentiated adenocarcinoma; Mod: moderately differentiated adenocarcinoma; Poor: poorly differentiated adenocarcinoma; ANED: alive with no evidence of disease; AWD: alive with disease; DOD: died of disease; ND: not done/measured; NS: not stated; NED: no evidence of disease; WNL: within normal limit; RN: reference number.

HCC. In addition, immunonegativity for p63, synaptophysin and chromogranin in the tumor cells help rule out squamous cell carcinoma and neuroendocrine carcinoma. Ki-67 highlights $80 \%$ of the tumor cells indicating a highly proliferating tumor.

Finding the primary tumor origin of HAC is crucial, and prompt and adequate treatment may improve outcomes of patients; but determining the tumor origin may become a challenge for physicians, especially when HAC was initially identified in the liver as metastatic lesions. In our presented case, interestingly, CDX2 is positive. CDX2 is a fairly specific marker of intestinal origin for adenocarcinomas, and can be used to determine the origin of metastatic adenocarcinoma as part of a panel [25]. SATB2 and CK20 are also positive, SATB2 is a biomarker for colorectal cancer (CRC), $85 \%$ of all CRC patients are positive for SATB2 and other cancer types rarely display SATB2 expression, SATB2 in combination with CK20 can identify over $95 \%$ of all colorectal carcinomas [26]. Based on the histologic features, immunoprofile, endoscopic and imaging findings, our case was diagnosed as liver with metastatic hepatoid colonic adenocarcinoma.

Because of this being a metastatic colorectal adenocarcinoma, we performed immunohistochemistry for MMR proteins (MLH1, MSH2, MSH6, and PMS2) and found intact expression of these proteins. Molecular testing of tumor by polymerase chain reaction (PCR) did not reveal any detectable MSI, indicating that DNA mismatch repair function within this tumor indeed was normal. NGS on the adenocarcinoma in the liver biopsy revealed the mutation in KRAS (c.38_39delGCinsAT p.G13D).

In terms of treatment, generally, radical surgery followed by systemic organ-specific adjuvant chemotherapy is considered the optimal treatment for HAC. A number of reported HAC cases underwent treatment using the regimen of FOLFOX (leucovorin, 5-fluorouracil, and oxaliplatin) or FOLFIRI (leucovorin, 5-fluorouracil, irinotecan). In a patient of HAC of the transverse colon without liver and lymph nodes metastasis, combination of a radical resection and adjuvant chemotherapy of FOLFOX-4 regimen had led to a recurrence-free survival of 27 months [6]. It is possible that since $\mathrm{HAC}$ is pathologically similar to HCC, agents used in primary HCC may be effective in HAC. Metzgeroth et al reported one case of a patient with primary HAC of the peritoneal cavity, of which the tumor tissue showed a strong activation of the epidermal growth factor receptor (EGFR) and the kinases ERK1 and AKT1 [27]. The patient was treated with sorafenib which had been known for its beneficial effects for HCC, and induced a temporary clinical stabilization but no significant reduction of the tumor mass and no substantial improvement of survival [27]. Interestingly, a patient with mismatch repair deficient HAC of the lung responded partially to anti-PD-L1 durvalumab immunotherapy despite no PD-L1expression, the author hypothesized that the therapeutic effect of immunotherapy can be due to the mismatch repair deficient status, as MMR-deficient tumors, with 
higher mutational load and increased expression of neoepitopes are known to be immunogenic tumors and potential good responders to immunotherapy [28]. Simmet et al reported two cases of patients with metastatic HAC of stomach successfully treated by chemotherapy with cisplatin and etoposide as firstline leading to complete response [29].

$\mathrm{HAC}$ is a very aggressive neoplasm with an unfavorable prognosis and a high proportion of metastases at the time of diagnosis, with the liver and lymph nodes being the most common metastatic sites. In this study, in the total of 18 cases of HAC [6-22], there were 12 patients with liver metastases and 11 patients with lymph nodes metastases. Twelve patients died of the disease within the first 12 months, and median overall survival time is 8 months, despite aggressive treatment with radical surgery followed by adjuvant chemotherapy. Due to the rarity of this adenocarcinoma, the cohort size of patients with HAC was not large enough, which may have resulted in some statistical bias.

In conclusion, we report an extremely rare case of metastatic colorectal adenocarcinoma with hepatoid features to the liver. Literature review shows colorectal HAC has a high rate of metastases at the time of diagnosis and dismal prognosis. Due to its rarity and aggressive behavior, it is important for pathologists to be aware of HAC in order to make a correct diagnosis, especially when this tumor has already metastasized to the liver, resembling HCC, both morphologically and clinically. Radical surgery followed by colorectal specific adjuvant chemotherapy is considered to be the optimal treatment for HAC.

\section{References}

1. Liu W, Zhang D, Tan SA, Liu X, Lai J. Sigmoid colon adenocarcinoma with isolated loss of PMS2 Presenting in a patient with synchronous prostate cancer with intact MMR: diagnosis and analysis of the family pedigree. Anticancer Res. 2018;38(8):4847-4852.

2. Bourreille J, Metayer P, Sauger F, Matray F, Fondimare A. [Existence of alpha feto protein during gastric-origin secondary cancer of the liver]. Presse Med. 1970;78(28):1277-1278.

3. Yang C, Sun L, Lai JZ, Zhou L, Liu Z, Xi Y, Tao Y, et al. Primary Hepatoid Carcinoma of the Pancreas: A Clinicopathological Study of 3 Cases With Review of Additional 31 Cases in the Literature. Int J Surg Pathol. 2018:1066896918783468.

4. Friedman P, Lai JP. Liver Metastasis of Urothelial Carcinoma with Hepatoid Features: An Unusual Morphological Finding. Anticancer Res. 2017;37(2):801-804.

5. Su JS, Chen YT, Wang RC, Wu CY, Lee SW, Lee TY. Clinicopathological characteristics in the differential diagnosis of hepatoid adenocarcinoma: a literature review. World J Gastroenterol. 2013;19(3):321-327.

6. Slotta JE, Jungling B, Kim YJ, Wagner M, Igna D, Schilling MK. Hepatoid adenocarcinoma of the transverse colon. Int J Colorectal Dis. 2012;27(7):989-991.

7. Nakajima T, Okazaki N, Morinaga S, Tsumuraya M, Shimosato Y, Saiki S. A case of alpha-fetoprotein-producing rectal carcinoma. Jpn J Clin Oncol. 1985;15(4):679-685.

8. Yu YY, Ogino T, Okada S. An alpha-fetoproteinproducing carcinoma of the rectum. Acta Pathol Jpn. 1992;42(9):684-687.

9. Sato Y, Sekine T, Ohwada S. Alpha-fetoprotein-producing rectal cancer: calculated tumor marker doubling time. J Surg Oncol. 1994;55(4):265-268.

10. Hocking GR, Shembrey M, Hay D, Ostor AG. Alphafetoprotein-producing adenocarcinoma of the sigmoid colon with possible hepatoid differentiation. Pathology. 1995;27(3):277-279.

11. Kato K, Matsuda M, Ingu A, Imai M, Kasai S, Mito M, Kobayashi T. Colon cancer with a high serum alphafetoprotein level. Am J Gastroenterol. 1996;91(5):10451046.

12. Taguchi J, Yano H, Sueda J, Yamaguchi R, Kojiro M, Shirouzu G, Hashimoto K. alpha-Fetoprotein-producing rectal carcinoma - a case report. Kurume Med J. 1997;44(4):339-348.

13. Kurihara K, Konishi F, Kanazawa K, Fujii T, Saito K. Alpha-fetoprotein-producing carcinoma of the colon: report of a case. Surg Today. 1997;27(5):453-456.

14. Ishikura H, Kishimoto T, Andachi H, Kakuta Y, Yoshiki T. Gastrointestinal hepatoid adenocarcinoma: venous permeation and mimicry of hepatocellular carcinoma, a report of four cases. Histopathology. 1997;31(1):47-54.

15. Yachida S, Fukushima N, Nakanishi Y, Akasu T, Kitamura H, Sakamoto M, Shimoda T. Alpha-fetoprotein-producing carcinoma of the colon: report of a case and review of the literature. Dis Colon Rectum. 2003;46(6):826-831.

16. Fu K, Kobayashi A, Saito N, Sano Y, Kato S, Ikematsu $\mathrm{H}$, Fujimori $\mathrm{T}$, et al. Alpha-fetoprotein-producing colon cancer with atypical bulky lymph node metastasis. World J Gastroenterol. 2006;12(47):7715-7716.

17. Orditura M, Lieto E, Ferraraccio F, De Cataldis G, Troiani T, Castellano P, Catalano G, et al. Hepatoid carcinoma colliding with a liposarcoma of the left colon serosa presenting as an abdominal mass. World J Surg Oncol. 2007;5:42.

18. Borgonovo G, Razzetta F, Assalino M, Varaldo E, Puglisi M, Ceppa P. Rectal hepatoid carcinoma with liver metastases in a patient affected by ulcerative colitis. Hepatobiliary Pancreat Dis Int. 2008;7(5):539-543.

19. Cappetta A, Bergamo F, Mescoli C, Lonardi S, Rugge M, Zagonel V. Hepatoid adenocarcinoma of the colon: what should we target? Pathol Oncol Res. 2012;18(1):93-96.

20. Chen Y, Schaeffer DF, Yoshida EM. Hepatoid adenocarcinoma of the colon in a patient with inflammatory bowel disease. World J Gastroenterol. 2014;20(35):1265712661.

21. Anzai H, Kazama S, Kiyomatsu T, Nishikawa T, Tanaka T, Tanaka J, Hata K, et al. Alpha-fetoprotein-producing early rectal carcinoma: a rare case report and review. World J Surg Oncol. 2015;13:180.

22. Armaghani A, Hernandez Gonzalo D, Daily K. Hepatoid adenocarcinoma of the colon. BMJ Case Rep. 2015;2015.

23. Yan BC, Gong C, Song J, Krausz T, Tretiakova M, Hyjek E, Al-Ahmadie H, et al. Arginase-1: a new immunohistochemical marker of hepatocytes and hepatocellular neo- 
plasms. Am J Surg Pathol. 2010;34(8):1147-1154.

24. Morrison C, Marsh W, Jr., Frankel WL. A comparison of CD10 to pCEA, MOC-31, and hepatocyte for the distinction of malignant tumors in the liver. Mod Pathol. 2002;15(12):1279-1287.

25. Park SY, Kim BH, Kim JH, Lee S, Kang GH. Panels of immunohistochemical markers help determine primary sites of metastatic adenocarcinoma. Arch Pathol Lab Med. 2007;131(10):1561-1567.

26. Magnusson K, de Wit M, Brennan DJ, Johnson LB, McGee SF, Lundberg E, Naicker K, et al. SATB2 in combination with cytokeratin 20 identifies over $95 \%$ of all colorectal carcinomas. Am J Surg Pathol. 2011;35(7):937-948.
27. Metzgeroth G, Strobel P, Baumbusch T, Reiter A, Hastka J. Hepatoid adenocarcinoma - review of the literature illustrated by a rare case originating in the peritoneal cavity. Onkologie. 2010;33(5):263-269.

28. Basse V, Schick U, Gueguen P, Le Marechal C, QuintinRoue I, Descourt R, Simon H, et al. A mismatch repairdeficient hepatoid adenocarcinoma of the lung responding to anti-PD-L1 Durvalumab therapy despite no PD-L1 expression. J Thorac Oncol. 2018;13(7):e120-e122.

29. Simmet V, Noblecourt M, Lizee T, Morvant B, Girault S, Soulie P, Capitain O. Chemotherapy of metastatic hepatoid adenocarcinoma: Literature review and two case reports with cisplatin etoposide. Oncol Lett. 2018;15(1):48-54. 